\title{
On the Total Vertex Irregularity Strength of Cycle Related Graphs and $H$ Graphs
}

\author{
Indra Rajasingh \\ Department of Adv. Sciences \\ V.I.T. University \\ Chennai, India
}

\author{
Bharati Rajan \\ Department of Mathematics \\ Loyola College \\ Chennai, India
}

\author{
V. Annamma \\ Department of Mathematics \\ L.N.Govenment College \\ Ponneri, India
}

\begin{abstract}
Let $G(V, E)$ be a simple graph. For a labeling $f: V \cup E \rightarrow\{1,2,3, \ldots, k\}$, the weight of a vertex $x$ is defined as $w t_{f}(x)=f(x)+\sum_{y \in N(x)} f(x)$ where $N(x)$ is the set of neighbours of $x . f$ is called a vertex irregular total k-labeling if for every pair of distinct vertices $x$ and $y w t_{f}(x) \neq w t_{f}(y)$

. The minimum $k$ for which the graph $G$ has a vertex irregular total $k$-labeling is called the total vertex irregularity strength of $G$ and is denoted by $t v s(G)$. In this paper we find the total vertex irregularity strength of cycle related graphs $H(n)$, $\operatorname{DHF}(n), F(n, 2)$ and obtain a bound for the total vertex irregularity strength of $H$ graphs $H(k)$.
\end{abstract}

\section{Keywords}

labeling, vertex irregular total $k$-labeling, total vertex irregularity strength (tvs).

\section{INTRODUCTION}

The area of graph theory has experienced a fast development during the last 60 years. Among all the different kinds of problems that appear while studying graph theory, one that has been growing strong during the last three decades is the area that studies labelings of graphs. This is not only due to its mathematical importance but also because of the wide range of applications arisisng from this area. Labeled graphs serve as useful models for a range of applications such as coding theory, x-ray, crystallography, radar, astronomy, circuit design, channel assignments of FM radio stations and communication network addressing[1].

A labeling of a graph is a function from the vertex set of a graph or the edge set of the graph or both to the set of natural numbers subject to certain conditions. All graphs considered in this paper are finite, simple, connected and undirected.

Let $G(V, E)$ be a simple graph. For a labeling $f: V \cup E$ $\rightarrow\{1,2,3, \ldots, k\}$, the weight of a vertex $x$ is defined as $w t_{f}(x)=f(x)+\sum_{y \in N(x)} f(x y)$, where $N(x)$ is the set of neighbours of $x . f$ is called a vertex irregular total $k$-labeling of $G$ if for every pair of distinct vertices $x$ and $y w t_{f}(x) \neq$ $w t_{f}(y)$. The minimum $k$ for which the graph $G$ has a vertex irregular total $k$-labeling is called the total vertex irregularity strength of $G$ and is denoted by $\operatorname{tvs}(G)$ [1]. Baca et al. [2] proved that $\operatorname{tvs}\left(C_{n}\right)=\lceil(n+2) / 3\rceil, n \geq 2 ; \operatorname{tvs}\left(K_{n}\right)=2$; $\operatorname{tvs}\left(K_{1, n}\right)=\lceil(n+1) / 2\rceil ; \operatorname{tvs}\left(C_{n} \times P_{2}\right)=\lceil(2 n+3) / 4\rceil$. Chunling et al. [3] showed that $\operatorname{tvs}\left(K_{p}\right)=2$ for $p \geq 2$ and $\operatorname{tvs}(P(n, k))=\lceil n / 2\rceil+1, \quad k \leq n / 2$ for the generalized Petersen graph $P(n, k)$. They also obtained the total vertex irregularity strength for ladder, Mobius ladder and Knodel graphs. For graphs with no isolated vertices, Pzrybylo [4] gave bounds for $t v s(G)$ in terms of the order, minimum and maximum degrees of $G$. For $d$-regular $(d>0)$ graphs, Przybylo [5] gave bounds for $\operatorname{tvs}(G)$ in terms of $d$ and the order of $G$. Ahmad et al. [6] determined the total vertex irregularity strength for five families of cubic plane graphs. They proved that for the circulant graphs $C_{n}(1,2), n \geq 5, t v s$ $\left(C_{n}(1,2)\right)=\lceil(n+4) / 2\rceil$. They also conjecture that for circulant graphs $C_{n}\left(a_{1}, a_{2}, a_{3} \ldots a_{m}\right)$ with degree at least 5 and $n \geq 5,1 \leq a_{i} \leq\left\lfloor\frac{n}{2}\right\rfloor, t v s\left(C_{n}\left(a_{1}, a_{2}, a_{3} \ldots a_{m}\right)\right)\lceil(n+r) /(1+r)\rceil$

In this paper we find the total vertex irregularity strength of cycle related graphs $H(n), D H F(n), F(n, 2)$ and obtain a bound for the total vertex irregularity strength of $H$ graphs $H(k)$.

\section{Total Vertex Irregularity Strength of $H(n)$}

In this section we obtain the total vertex irregularity strength of $H(n)$

Definition 2.1 [7]: $H(n)$ for $n \geq 4$ is defined as $V(H(n))=$ $\left\{v_{0}, v_{1}, v_{2}, \ldots, v_{n-1}\right\}$ and $E(H(n))=\left\{\left(v_{i}, v_{i+1}\right), 0 \leq i \leq n-2\right\} \cup$ $\left\{\left(v_{0}, v_{n / 2}\right), \quad\left(v_{0}, v_{n-1}\right)\right\} \cup\left\{\left(v_{i} v_{n} \quad i \quad 1 \leq i \leq n / 2-1\right\} \quad n\right.$ being an even integer. We name the edge $\left(v_{0}, v_{n / 2}\right)$ as chord and $\left(v_{i}, v_{n-i}\right), \quad 1 \leq i \leq n / 2-1$ as parallel chords. $H(n)$ is also a 1-fault-tolerant Hamiltonian graph. See Figure 1(a). 

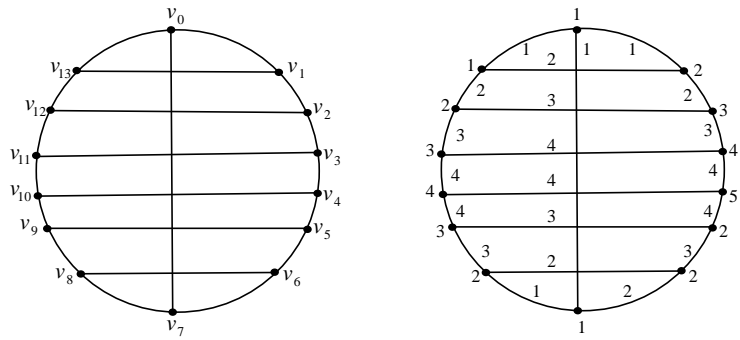

Figure 1: (a) $H(14)$; (b) Labeled $H(14)$

The following Theorem is the key result used for proving Theorems 2.2 and 5.1.

Theorem 2.1 [4]: Let $G$ be an $r$-regular graph on $n$ vertices.

Then $t v s(G) \geq\left\lceil\frac{n+r}{r+1}\right\rceil$.

Theorem 2.2: Let $G$ be $H(n)$. Then $\operatorname{tvs}(H(n))=\left\lceil\frac{n+3}{4}\right\rceil$, $n \geq 4$.

Proof: We give an algorithm to label the vertices and edges of $H(n)$ using exactly $\left\lceil\frac{n+3}{4}\right\rceil$ labels.

Let $k$ denote the number of chords. Then clearly $k=n / 2-1$. Algorithm : tvs $(H(n))$

Input: $H(n), n \geq 4$

Case (1) : $k$ odd.

(i) Vertices $v_{0}$ and $v_{n / 2}$ receive label 1.

(ii) Vertex adjacent to $v_{0}$ in the anticlockwise direction receives label 1 and the $\left\lceil\frac{k}{2}\right\rceil-1$ consecutive vertices in the same direction receive labels $2,3,4, \ldots,\left\lceil\frac{n+3}{4}\right\rceil-1$.

(iii) Vertex adjacent to $v_{0}$ in the clockwise direction receives label 2 and the $\left\lceil\frac{k}{2}\right]-1$ consecutive vertices in the same direction receive label 3,4 , $5 \ldots,\left\lceil\frac{n+3}{4}\right\rceil$.

(iv) Vertex adjacent to $v_{n / 2}$ in the clockwise direction receives label 2 and the $\left\lceil\frac{k}{2}\right\rceil-2$ consecutive vertices in the same direction receives label $3,4, \ldots,\left\lceil\frac{n+3}{4}\right\rceil-1$.

(v) Vertex adjacent to $v_{n / 2}$ in the anticlockwise direction receives label 2 and the $\left\lceil\frac{k}{2}\right\rceil-2$ consecutive vertices in the same direction receive labels $2,3,4, \ldots,\left\lceil\frac{n+3}{4}\right\rceil-2$.

Edge incident at $v_{0}$ in the anticlockwise direction receives label 1 and the $\left\lceil\frac{k}{2}\right\rceil-1$ consecutive edges in the same direction receive labels $2,3,4, \ldots,\left\lceil\frac{n+3}{4}\right\rceil-1$.

(vii) Edge incident at $v_{0}$ in the clockwise direction receives label 1 and the $\left\lceil\frac{k}{2}\right\rceil-1$ consecutive edges in the same direction receive labels 2, 3, $4, \ldots,\left\lceil\frac{n+3}{4}\right\rceil-1$.

Edge incident at $v_{n / 2}$ in the clockwise direction receives label 1 and the $\left\lceil\frac{k}{2}\right\rceil-1$ consecutive edges in the same direction receive labels $3,4,5, \ldots,\left\lceil\frac{n+3}{4}\right\rceil$.

(ix) Edge incident at $v_{n / 2}$ in the anticlockwise direction receives label as 2 and the $\left\lceil\frac{k}{2}\right\rceil-1$ consecutive edges in the same direction receive labels $3,4,5, \ldots,\left[\frac{n+3}{4}\right]$.

(x) Chord $\left(v_{0}, v_{n / 2}\right)$ receives label 1.

(xi) Parallel chord below $v_{0}$ receives label 2 and the $\left\lceil\frac{k}{2}\right\rceil-1$ successive parallel chords receive labels $3,4,5, \ldots,\left\lceil\frac{n+3}{4}\right\rceil$.

(xii) Parallel chord above $v_{n / 2}$ receives label 2 and the $\left\lceil\frac{k}{2}\right\rceil-2$ successive parallel chords receive labels $3,4,5, \ldots,\left\lceil\frac{n+3}{4}\right\rceil-1$. See Figure 1(b).

Case (2): $k$ even.

(i) Vertices $v_{0}$ and $v_{n / 2}$ receive label 1.

(ii) Vertex adjacent to $v_{0}$ in the anticlockwise receives label 1 and the $\left\lceil\frac{k+1}{2}\right\rceil-1$ consecutive vertices in the same direction receive labels $2,3,4, \ldots,\left\lceil\frac{n+3}{4}\right\rceil-1$.

(iii) Vertex adjacent to $v_{0}$ in the clockwise direction receives label 2 and the $\left\lceil\frac{k+1}{2}\right\rceil-1$ consecutive vertices in the same direction receive labels $3,4,5, \ldots,\left\lceil\frac{n+3}{4}\right\rceil$. 
(iv) Vertex adjacent to $v_{n / 2}$ in the clockwise direction receives label 2 and the $\left\lceil\frac{k+1}{2}\right\rceil-3$ consecutive vertices in the same direction receive labels $3,4,5, \ldots,\left[\frac{n+3}{4}\right]-2$.

(v) Vertex adjacent to $v_{n / 2}$ in the anticlockwise direction receives label 2 and the $\left\lceil\frac{k+1}{2}\right\rceil-3$ consecutive vertices in the same direction receive labels $2,3,4, \ldots,\left\lceil\frac{n+3}{4}\right\rceil-3$.

(vi) Edge incident at $v_{0}$ in the anticlockwise direction receives label 1 and the $\left\lceil\frac{k+1}{2}\right\rceil-1$ consecutive edges in the same direction receive labels $2,3,4, \ldots,\left\lceil\frac{n+3}{4}\right\rceil-1$.

(vii) Edge incident at $v_{0}$ in the clockwise direction receives label 1 and the $\left\lceil\frac{k+1}{2}\right\rceil-1$ consecutive edges in the same direction receive labels $3,4,5, \ldots,\left\lceil\frac{n+3}{4}\right\rceil-1$.

(viii) Edge incident at $v_{n / 2}$ in the clockwise direction receives label 1 and the $\left\lceil\frac{k+1}{2}\right\rceil-2$ consecutive edges in the same direction receive labels $3,4,5, \ldots,\left\lceil\frac{n+3}{4}\right\rceil-1$.

(ix) Edge incident at $v_{n / 2}$ in the anticlockwise direction receives label 2 and the $\left\lceil\frac{k+1}{2}\right\rceil-2$ consecutive edges in the same direction receive labels $3,4,5, \ldots,\left\lceil\frac{n+3}{4}\right\rceil$.

(x) Chord $\left(v_{0}, v_{n / 2}\right)$ receives label 1.

(xi) Parallel chord below $v_{0}$ receives label 2 and the $\left\lceil\frac{k+1}{2}\right\rceil-2$ successive parallel chords receive labels $3,4,5, \ldots,\left\lceil\frac{n+3}{4}\right\rceil-1$.

(xii) Parallel chord above $v_{n / 2}$ receives label 2 and the $\left\lceil\frac{k+1}{2}\right\rceil-1 \quad$ successive parallel chords receive labels $3,4,5, \ldots,\left\lceil\frac{n+3}{4}\right\rceil-1$. See Figure 1(b).

Output: $t v s(H(n))=\left\lceil\frac{n+3}{4}\right\rceil, n \geq 4$.

Proof of Correctness : The weights of the vertices successively attain values $4,5,6, \ldots, n+3$ when the vertices and edges of $H(n)$ receive labels from the set $\left\{1,2,3, \ldots,\left\lceil\frac{n+3}{4}\right\rceil\right\}$. Hence the weights of the vertices are distinct. The maximum label used is $\left\lceil\frac{n+3}{4}\right\rceil$. Hence by Theorem $2.1 \operatorname{tvs}(H(n))=\left\lceil\frac{n+3}{4}\right\rceil, n \geq 4$.

\section{Total Vertex Irregularity of Double Headed Circular Fan $D H F(n)$}

In this section we obtain the total vertex irregularity strength of double headed circular fan $\operatorname{DHF}(n)$.

Definition 3.1 [8]: Let $C: v_{1} v_{2} v_{3} \ldots v_{\mathrm{n}} v_{1}$ be a cycle on $n$ vertices. For $v_{i} \in V(C)$, the graph obtained by adding edges $\left(v_{i}, u\right), i=1,2,3, \ldots, n-3$ and $\left(v_{i}, v\right), i=n-2, n-1, n$ to C is called double headed circular fan. It is denoted by $\operatorname{DHF}(n)$. See Figure 2 (a).
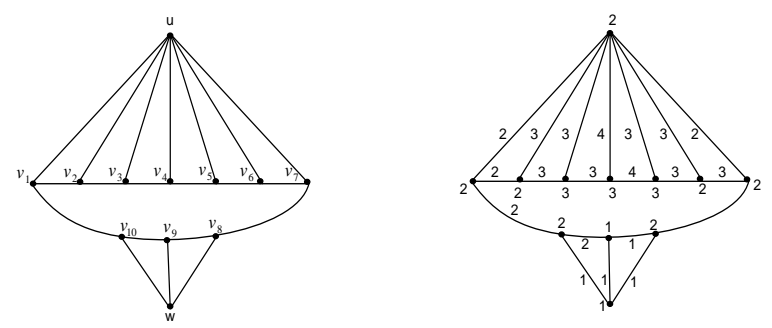

Figure 2: (a) $D H F(10)$; (b) Labeled $\operatorname{DHF}(10)$

Theorem 3.1 [4]: Let $G$ be a graph with no isolated vertices or edges. Then $\operatorname{tvs}(G) \leq n-1-\left\lfloor\frac{n-2}{\Delta+1}\right\rfloor$.

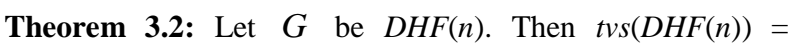
$\left\lceil\frac{n+4}{4}\right\rceil, n \geq 4$.

Proof: We give an algorithm to label the vertices, edges of $\operatorname{DHF}(n)$ respectively.

Algorithm A: tvs $(D H F(n))$

Input: Vertices of $(D H F(n)), n \geq 4$

Step 1: Vertices $v_{n-2}, v_{n}$ receive label 2 .

Step 2: Vertices $w, v_{n-1}$ receive label 1 .

Let $k=n-3, n \geq 7$.

Case (1): $k \equiv 0(\bmod 4)$

(i) Vertices $v_{1}, v_{2}, v_{3}, \ldots, v_{k / 2-1}, v_{k / 2}$ receive labels in the order $2,2,3,3,4,4, \ldots,\left\lceil\frac{n+4}{4}\right\rceil-2$, $\left\lceil\frac{n+4}{4}\right\rceil-2,\left\lceil\frac{n+4}{4}\right\rceil-1,\left\lceil\frac{n+4}{4}\right\rceil-1$. 
(ii) Vertices $v_{k / 2+1}, v_{k / 2+2}, \ldots, v_{k-1}, v_{k}$ receive labels in the order $\left\lceil\frac{n+4}{4}\right\rceil-1,\left\lceil\frac{n+4}{4}\right\rceil-1,\left\lceil\frac{n+4}{4}\right\rceil-2$, $\left\lceil\frac{n+4}{4}\right\rceil-2, \ldots, 3,3,2,2$.

\section{Case (2): $k \equiv 1(\bmod 4)$}

(i) Vertices $v_{1}, v_{2}, v_{3}, \ldots, v_{\lceil k / 2\rceil-2}, v_{\lceil k / 2\rceil-1}$ receive labels in the order $2,2,3,3,4,4, \ldots,\left\lceil\frac{n+4}{4}\right\rceil-2$, $\left\lceil\frac{n+4}{4}\right\rceil-2, .\left\lceil\frac{n+4}{4}\right\rceil-1,\left\lceil\frac{n+4}{4}\right\rceil-1$.

(ii) $\quad$ Vertex $v_{\lceil k / 2\rceil}$ receives label $\left\lceil\frac{n+4}{4}\right\rceil$.

(iii) Vertices $v_{\lceil k / 2\rceil+1}, v_{\lceil k / 2\rceil+2}, \ldots, v_{k-1}, v_{k}$ receive labels in the order $\left\lceil\frac{n+4}{4}\right\rceil-1,\left\lceil\frac{n+4}{4}\right\rceil-1$.. $\left\lceil\frac{n+4}{4}\right\rceil-2,\left\lceil\frac{n+4}{4}\right\rceil-2, \ldots ., 3,3,2,2$.

Case (3): $k \equiv 2(\bmod 4)$

(i) Vertices $v_{1}, v_{2}, v_{3}, \ldots, v_{k / 2}, v_{k / 2+1}$ receive labels in the order $2,2,3,3,4,4, \ldots,\left\lceil\frac{n+4}{4}\right\rceil-2$, $\left\lceil\frac{n+4}{4}\right\rceil-2,\left\lceil\frac{n+4}{4}\right\rceil-1,\left\lceil\frac{n+4}{4}\right\rceil-1$.

(ii) Vertices $v_{k / 2+2}, v_{k / 2+3}, \ldots, v_{k-1}, v_{k}$ receive labels in the order $\left\lceil\frac{n+4}{4}\right\rceil-2,\left\lceil\frac{n+4}{4}\right\rceil-2,\left\lceil\frac{n+4}{4}\right\rceil-3$, $\left\lceil\frac{n+4}{4}\right\rceil-3, \ldots, 3,3,2,2$.

Case (4): $k \equiv 3(\bmod 4)$

(i) Vertices $v_{1}, v_{2}, v_{3}, \ldots, v_{\lceil k / 2\rceil-1}, v_{\lceil k / 2\rceil}$ receive labels in the order $2,2,3,3,4,4, \ldots,\left\lceil\frac{n+4}{4}\right\rceil-2$, $\left\lceil\frac{n+4}{4}\right\rceil-2,\left\lceil\frac{n+4}{4}\right\rceil-3,\left\lceil\frac{n+4}{4}\right\rceil-3$.

(ii) $\quad$ Vertex $v_{\lceil k / 2\rceil+1}$ receives label $\left\lceil\frac{n+4}{4}\right\rceil-1$.

(iii) Vertices $v_{\lceil k / 2\rceil+2}, v_{\lceil k / 2\rceil+3}, \ldots, v_{k-1}, v_{k}$ receive labels in the order $\left\lceil\frac{n+4}{4}\right\rceil-2,\left\lceil\frac{n+4}{4}\right\rceil-2$, $\left\lceil\frac{n+4}{4}\right\rceil-3,\left\lceil\frac{n+4}{4}\right\rceil-3, \ldots ., 3,3,2,2$.

We give an algorithm to label the edges of $\operatorname{DHF}(n)$ Let $e_{i}=\left(x_{\mathrm{i}}, x_{\mathrm{i}+1}\right), \quad 1 \leq i \leq n-4$ and $g_{j}=\left(u, x_{\mathrm{i}}\right), 1 \leq i$ $\leq n-3$. .

Algorithm B: tvs $(D H F(n))$

Input: Edges of $(\operatorname{DHF}(n)), n \geq 4$

Step 1: Edges $\left(w, v_{\mathrm{i}}\right), n-2 \leq i \leq n$ and edge $\left(v_{\mathrm{n}-2}, v_{\mathrm{n}-1}\right)$ receive label 1 .
Step 2: Edges $\left(v_{\mathrm{n}-1}, v_{\mathrm{n}}\right),\left(v_{\mathrm{n}}, v_{1}\right),\left(v_{\mathrm{n}-3}, v_{\mathrm{n}-2}\right)$ receive label 2. Step3: $e_{1}, g_{1}, g_{n-3}$ receive label 2

Case (1): $i \equiv 0(\bmod 4), j \equiv 1(\bmod 4)$.

(i) Edges $e_{2}, e_{3}, \ldots, e_{i / 2-2}, e_{i / 2-1}$ receive labels in the order $3,3,4,4, \ldots,\left\lceil\frac{n+4}{4}\right\rceil-2,\left\lceil\frac{n+4}{4}\right\rceil-2$, $\left\lceil\frac{n+4}{4}\right\rceil-1,\left\lceil\frac{n+4}{4}\right\rceil-1$.

Edge $e_{i / 2}$ receives label $\left\lceil\frac{n+4}{4}\right\rceil$.

Edges $e_{i / 2+1}, e_{i / 2+2}, \ldots, e_{i-1}, e_{i}$ receive labels in the order $\left\lceil\frac{n+4}{4}\right\rceil,\left\lceil\frac{n+4}{4}\right\rceil,\left\lceil\frac{n+4}{4}\right\rceil-1,,\left\lceil\frac{n+4}{4}\right\rceil$ $-1, \ldots, 4,4,3,3$.

(iv) Edges $g_{2}, g_{3}, \ldots, g_{\lceil j / 2\rceil-1}, g_{\lceil j / 2\rceil}$ receive labels in the order $3,3,4,4, \ldots,\left\lceil\frac{n+4}{4}\right\rceil-1,\left\lceil\frac{n+4}{4}\right\rceil-1$, $\left\lceil\frac{n+4}{4}\right\rceil,\left\lceil\frac{n+4}{4}\right\rceil$

(v) Edge $g_{\lceil j / 2\rceil}$ receives label $\left\lceil\frac{n+4}{4}\right\rceil$.

(vi) Edges $g_{\lceil j / 2\rceil+1}, g_{\lceil j / 2\rceil+2}, \ldots, g_{j-1}, g_{j}$ receive labels in the order $\left\lceil\frac{n+4}{4}\right\rceil-1,\left\lceil\frac{n+4}{4}\right\rceil-1,\left\lceil\frac{n+4}{4}\right\rceil-2$, $\left\lceil\frac{n+4}{4}\right\rceil-2, \ldots, 3,3,2,2$.

Case (2): $i \equiv 1(\bmod 4), j \equiv 2(\bmod 4)$

(i) Edges $e_{2}, e_{3}, \ldots, e_{\lceil i / 2\rceil-1}, e_{\lceil i / 2\rceil}$ receive labels in the order $3,3,4,4, \ldots,\left\lceil\frac{n+4}{4}\right\rceil-2,\left\lceil\frac{n+4}{4}\right\rceil-2$, $\left\lceil\frac{n+4}{4}\right\rceil-1,\left\lceil\frac{n+4}{4}\right\rceil-1$.

(ii) Edges $e_{\lceil i / 2\rceil+1}, e_{\lceil i / 2\rceil+2}, \ldots, e_{i-1}, e_{i}$ receive labels in the order $\left\lceil\frac{n+4}{4}\right\rceil-1,\left\lceil\frac{n+4}{4}\right\rceil-1,\left\lceil\frac{n+4}{4}\right\rceil-2$, $\ldots, 4,4,3,3$.

(iii) Edges $g_{2}, g_{3}, \ldots, g_{j / 2-1}, g_{j / 2}$ receive labels in the order $3,3,4,4, \ldots,\left\lceil\frac{n+4}{4}\right\rceil-2,\left\lceil\frac{n+4}{4}\right\rceil-2$, $\left\lceil\frac{n+4}{4}\right\rceil-1,,\left\lceil\frac{n+4}{4}\right\rceil-1$.

(iv) Edges $g_{\lceil j / 2\rceil^{+1}}$ and $g_{\lceil j / 2\rceil^{+2}}$ receive label $\left\lceil\frac{n+4}{4}\right\rceil-1$.

(v) Edges $g_{j / 2+3}, g_{j / 2+4}, \ldots, g_{j-1}, g_{j}$ receive labels in the order $\left\lceil\frac{n+4}{4}\right\rceil-2,\left\lceil\frac{n+4}{4}\right\rceil-2,\left\lceil\frac{n+4}{4}\right\rceil-3$, $\left\lceil\frac{n+4}{4}\right\rceil-3, \ldots, 3,3,2,2$. 
Case (3): $i \equiv 2(\bmod 4), j \equiv 3(\bmod 4)$.

(i) Edges $e_{2}, e_{3}, \ldots, e_{i / 2-1}, e_{i / 2}$ receive labels in the order $3,3,4,4, \ldots,\left\lceil\frac{n+4}{4}\right\rceil-2,\left\lceil\frac{n+4}{4}\right\rceil-2$, $\left\lceil\frac{n+4}{4}\right\rceil-1,\left\lceil\frac{n+4}{4}\right\rceil-1$.

(ii) Edge $e_{i / 2+1}$ receives label $\left\lceil\frac{n+4}{4}\right\rceil$.

(iii) Edges $e_{i / 2+2}, e_{i / 2+3}, \ldots, e_{i-1}, e_{i}$ receive labels in the order $\left\lceil\frac{n+4}{4}\right\rceil-1,,\left\lceil\frac{n+4}{4}\right\rceil-1, \quad\left\lceil\frac{n+4}{4}\right\rceil-2$, $\left\lceil\frac{n+4}{4}\right\rceil-2, \ldots, 4,4,3,3$.

(iv) Edges $g_{2}, g_{3}, \ldots, g_{\lceil j / 2\rceil}, g_{\lceil j / 2\rceil+1}$ receive labels in the order $3,3,4,4, \ldots,\left\lceil\frac{n+4}{4}\right\rceil-1,\left\lceil\frac{n+4}{4}\right\rceil-1$, $\left\lceil\frac{n+4}{4}\right\rceil,\left\lceil\frac{n+4}{4}\right\rceil$

(v) Edges $g_{\lceil j / 2\rceil+2}, g_{\lceil j / 2\rceil+3}, \ldots, g_{j-1}, g_{j}$ receive labels in the order $\left\lceil\frac{n+4}{4}\right\rceil-1,\left\lceil\frac{n+4}{4}\right\rceil-1,\left\lceil\frac{n+4}{4}\right\rceil-2$, $\left\lceil\frac{n+4}{4}\right\rceil-2, \ldots, 4,4,3,3$.

Case (4): $i \equiv 3(\bmod 4), j \equiv 0(\bmod 4)$

(i) Edges $e_{2}, e_{3}, \ldots, e_{\lceil i / 2\rceil}, e_{\lceil i / 2\rceil+1}$ receive labels in the order $3,3,4,4, \ldots,\left\lceil\frac{n+4}{4}\right\rceil-1,\left\lceil\frac{n+4}{4}\right\rceil-1$, $\left\lceil\frac{n+4}{4}\right\rceil,\left\lceil\frac{n+4}{4}\right\rceil$.

(ii) Edges $e_{\lceil i / 2\rceil+2}, e_{\lceil i / 2\rceil+3}, \ldots, e_{i-1}, e_{i}$ receive labels in the order $\left\lceil\frac{n+4}{4}\right\rceil-1,\left\lceil\frac{n+4}{4}\right\rceil-1,\left\lceil\frac{n+4}{4}\right\rceil-2$, $\ldots, 4,4,3,3$.

(iii) Edges $g_{2}, g_{3}, \ldots, g_{j / 2}, g_{j / 2+1}$ receive labels in the order $3,3,4,4, \ldots,\left\lceil\frac{n+4}{4}\right\rceil-1,\left\lceil\frac{n+4}{4}\right\rceil-1$, $\left\lceil\frac{n+4}{4}\right\rceil,\left\lceil\frac{n+4}{4}\right\rceil$

(iv) Edges $g_{j / 2+2}, g_{j / 2+3}, \ldots, g_{j-1}, g_{j}$ receive labels in the order $\left\lceil\frac{n+4}{4}\right\rceil-1,\left\lceil\frac{n+4}{4}\right\rceil-1,\left\lceil\frac{n+4}{4}\right\rceil-2$, $\left\lceil\frac{n+4}{4}\right\rceil-2, \ldots, 4,4,3,3$.

Output: $\operatorname{tvs}(\operatorname{DHF}(n))=\left\lceil\frac{n+4}{4}\right\rceil, n \geq 4$.
Proof of Correctness : The weights of the vertices on the cycle and the weight of the vertex $w$ successively attain values $4,5,6, \ldots, n+4$ when the vertices and edges of $\operatorname{DHF}(n)$ receive labels from the set $\left\{1,2,3, \ldots,\left\lceil\frac{n+4}{4}\right\rceil\right\}$. Also $w t(u)>n$ +4 . Hence the weights of the vertices are distinct. The maximum label used is $\left\lceil\frac{n+4}{4}\right\rceil$. Hence by theorem 3.1 $\operatorname{tvs}(\operatorname{DHF}(n))=\left\lceil\frac{n+4}{4}\right\rceil, n \geq 4$.

\section{Total vertex irregularity strength of circular fan with two chords $F(n, 2)$}

In this section we obtain the total vertex irregularity strength of circular fan with two chords $F(n, 2)$.

Definition 4.1 [8]: Let C: $x_{1} x_{2} x_{3} \ldots x_{n} x_{1}$ be a cycle on $n$ vertices. Let $u$ be a new vertex. The graph obtained by adding edges $\left(u, x_{i}\right), i=1,2,3 \ldots n-4$ to $C$ and chords $\left(x_{n-1}, x_{n-3}\right)$ and $\left(x_{n-2}, x_{n}\right)$ is called a circular fan with two chords and is denoted by $F(n, 2)$. See Figure 3(a).
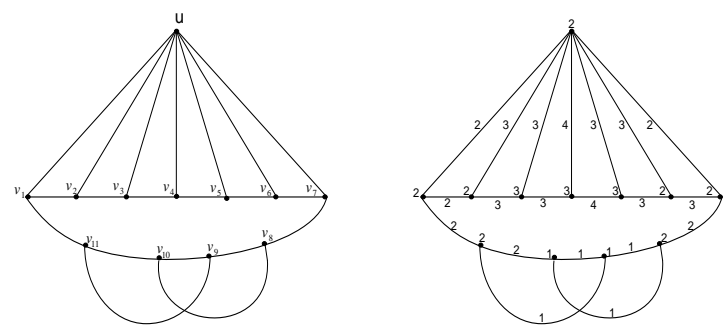

Figure 3: (a) $F(11,2)$; (b) Labeled $F(11,2)$

Theorem 4.1: $t v s(F(n, 2))=\left\lceil\frac{n+4}{4}\right\rceil, n \geq 4$.

Proof is similar to theorem 3.1.

\section{Total vertex irregularity strength of $H$ Graphs $H(k)$}

In this section we obtain the total vertex irregularity strength of $H$ graphs $H(k)$.

Definition 5.1 [9]: An $H$ graph $H(k), k$ is the number of copies of $H$ is a 3-regular graph with vertex set $V=\{(i, j), 1 \leq i \leq$ $3 ; 1 \leq j \leq 2 k\}$ and edge set $E=\{((i, j),(i, j+1)), i=1,3,1$ $\leq j \leq 2 k-1\} \cup\{((2, j),(2, j+1)), j$ odd, $1 \leq j \leq 2 k-1\}$ $\cup\{((1,1),(1,2 k)),((3,1),(3,2 k))\} \cup\{((i, j),(i+1, j), i=1$, $2,1 \leq j \leq 2 k\}$. An $H$ graph $H(k)$ has $6 k$ vertices and $9 k$ edges. See Figure 4(a). 

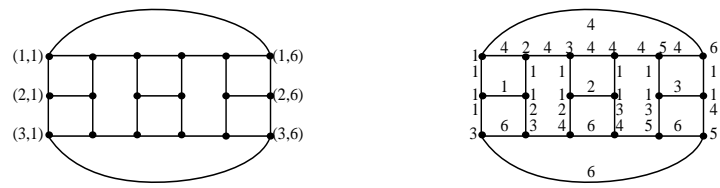

Figure 4: (a) H(3); (b) Labeled $H(3)$

Remark 1: $t v s(H(k))=4$ for $k=2$, tvs $(H(k))=6$ for $k=3$.

Theorem 5.1: $\left\lceil\frac{6 k+3}{4}\right\rceil \leq t v s(H(k)) \leq 2 k$, for $k \geq 4$.

Proof: The lower bound follows from theorem 2.1.

We give an algorithm to label the vertices and edges of $H(k)$.

Algorithm : tvs $(H(k))$

Input: $H(k), k \geq 4$

(i) Vertices $(1, i), 1 \leq i \leq 2 k$ receive label $i$ from left to right.

(ii) Vertices $(2, i), 1 \leq i \leq 2 k$ receive label 1 .

(iii) Vertices $(3,2 i-1),(3,2 i), 1 \leq i \leq k$ receive label $i+2$.

(iv) Each edge between the first row and the second row receives label 1 .

(v) The remaining edges induced by the vertices in the first row receive label $k+1$.

(vi) Edges induced by the vertices $(2,2 i-1), 1 \leq i$ $\leq k$, along the second row receive label $i$.

(vii) Edges below the second row induced by the vertices $(2, i), 1 \leq i \leq 2 k$ receive the label $\left\lceil\frac{i+1}{2}\right\rceil$.

(viii) Edges induced by the vertices $(3, i), 1 \leq i \leq$ $2 k$ receives the label $2 k$. See Figure $3(\mathrm{~b})$.

Output: $\left\lceil\frac{6 k+3}{4}\right\rceil \leq t v s(H(k)) \leq 2 k,, k \geq 4$.

Proof of Correctness : The weights of the vertices successively attain values $4,5,6, \ldots, n+3$ when the vertices and edges of $H(k)$ receive labels from the set $\{1,2,3, \ldots$, $2 k$ \}. Hence this labeling provides an upper bound on $t v s(H(k))$. Thus $t v s(H(k)) \leq 2 k$.

\section{CONCLUSION}

The concept of vertex labeling is likely to have good application in laying roads connecting important cities. The weight of each edge may be the cost incurred for constructing the roads between two cities. The minimum weight gives the minimum total cost of construction of roads connecting the cities. In this paper we have determined the total vertex irregularity strength of cycle related graphs $H(n)$, $\operatorname{DHF}(n), F(n, 2)$ and obtained a bound for the total vertex irregularity strength of $H$ graphs. Total vertex irregular $k$ labeling for networks like hexagonal network, butterfly network and benes network is under investigation.

\section{REFERENCES}

[1] Joseph A.Gallian, 2010. A Dynamic Survey of Graph Labeling. The Electronic Journal of Combinatorics.

[2] S. Baca, J. Jenrol, M. Miller \& J. Ryan, 2007. On Irregular Total Labelings. Discrete Mathematics, vol. 307, pp. 1378-1388.

[3] Chunling. Irregular Total Labelings of some Families of Graph. Indian Journal of Pure and Applied Mathematics, to appear.

[4] J.Przybylo, 2008. Irregularity Strength of Regular Graphs. Electronic Journal of Combinatorics 15.

[5] J.Przybylo, 2008/2009. Linear Bound on the Irregularity Strength and Total Vertex Irregularity Strength of Graphs. SIAM Journal of Discrete Mathematics, 23, 511516.

[6] Ahmaad, S.Ahtsham, Imran and A.Q.Baig. Vertex Irregular Labelings of Cubic Graphs. reprint.

[7] Wang, J.-J., Hung, C.-N., Tan, J.J.M., Hsu, L.-H. and Sung T-Y, 2000. Construction Schemes for Fault Tolerant HamiltonianGraphs. Networks, 35, 233.

[8] Indra Rajasingh, Bharathi Rajan and Joice Punitha, 2007. Kernel in Cycle Related Graphs, Proceedings of the International Conference on Mathematics and Computer Science 275-278.

[9] Indra Rajasingh, Bharathi Rajan and Helda Mercy, 2009. Exact Wirelength of $H$ Graphs on Path, Proceedings of the International Conference on Mathematics and Computer Science 275-278. 